Editorial

\title{
Acknowledgment to Reviewers of Viruses in 2020
}

\author{
Viruses Editorial Office
}

Citation: Viruses Editorial Office. Acknowledgment to Reviewers of Viruses in 2020. Viruses 2021, 13, 98 https://doi.org/10.3390/v13010098

Published: 12 January 2021

Publisher's Note: MDPI stays neutral with regard to jurisdictional claims in published maps and institutional affiliations.

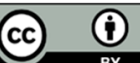

Copyright: $\odot 2021$ by the author. Licensee MDPI, Basel, Switzerland. This article is an open access article distributed under the terms and conditions of the Creative Commons Attribution (CC BY) license (http://creativecommons.org/licenses/by/4.0/).

MDPI AG, St. Alban-Anlage 66, 4052 Basel, Switzerland

Peer review is the driving force of journal development, and reviewers are gatekeepers who ensure that Viruses maintains its standards for the high quality of its published papers. Thanks to the cooperation of our reviewers, in 2020, the median time to first decision was 16 days and the median time to publication was 35 days. The editors would like to express their sincere gratitude to the following reviewers for their precious time and dedication, regardless of whether the papers were finally published:

A. McGargill, Maureen

Abdallah, Ali

Abdel Hakeem, Mohamed

Abdel-Mohsen, Mohamed T.

Abdel-Whab, El-Sayed

Abdelwhab, El-Sayed M

Abedon, Stephen

Abergel, Chantal

Abolnik, Celia

ABOUL-ATA, Aboul-Ata

Abreu, Cândida

Accardi, Luisa

Accotto, Gian Paolo

Acharya, Priyamvada

Ackermann, Mathias

Adams, Ian

Adams, Sandra D.

Adegbola, Raphael

Ades, Tony

Adkar-Purushothama, Charith Raj

Adriaenssens, Evelien

Aggarwal, Megha

Aguilar-Carreno, Hector

Aherfi, Sarah

Ahmad, Asrar

Aiken, Christopher

Airenne, Kari

Akatsuki, Saito

Akhmetzhanov, Andrei R.

Akimov, Sergei

Akiyama, Hisashi

Alabi, Olufemi

Alam, Samina

Alazem, Mazen

Albarnaz, J. D.
Albrecht, Randy

Alejo, Alí

Alekseev, Konstantin

Alfieri, Caroline

Ali Zaidi, Shan

Allard, Benoit

Alley, Maurice R.

Allison, Andrew

Allison, Heather E.

Almeida, Gabriel

Almendral, José

Alonso, Covadonga

Alonso, Marta

Alphonse, Sebastien

Altamura, Gennaro

Altan-Bonnet, Nihal

Aly, Hussein

Alymova, Irina V.

Amarasiri, Mohan

Amarilla, Alberto A.

Ambagala, Aruna

Amiri, Esmaeil

Amoutzias, Grigoris

An, Dong Sung

An, Dong-Jun

Anany, Hany

Anaya-López, Jose Luis

Anda, Baicus

Anderson, Danielle

Anderson, John

Andersson, Bjorn

Ando, Sugihiro

András, Ibolya

Andreoni, Kenneth A.

Angelova, Assia L. 
Anis, Eman

Ansaldi, Mireille

Anticoli, Simona

Anton, Halina

Antonyuk, Svetlana

Antson, Fred

Antunes, Francisco José Nunes

Aparicio, Frederic

Apolonia, Luis

Aquaro, Stefano

Arakelyan, Anush

Aralaguppe, Shambhu G.

Aranda, Miguel A.

Aranday-Cortes, Elihu

Araniciu, Cătălin

Arbuthnot, Patrick

Arduino, Roberto C.

Arens, Ramon

Argaw, Takele

Arhel, Nathalie J.

Arias, Armando

Arias, Carolina

Arien, Kevin K.

Arii, Jun

Arkin, Isaiah

Aronson, Judy

Arriagada, Gloria

Asante, Emmanuel

Asgari, Sassan

Ashour, Hossam

Aston, Emily J.

Atanasova, Kalina

Atkinson, Sarah C.

Attias, Joseph

Atwood, Walter

Audet, Jonathan

Audsley, Michelle

Auvinen, Eeva

Avinoam, Ori

Avrani, Sarit

Awasthi, Sita

Azab, Walid

Azam, Aa Haeruman

Azar, Sasha R.

Azzi, Alberta

Baaijens, Jasmijn

Babiuk, Shawn

Babkin, Igor V.

Bacharach, Eran

Bachmann, Martin F

Bagasra, Omar
Bagchi, Parikshit

Bagiu, Iulia-Cristina

Baigent, Sue

Bailey, Adam Lee

Bailey, Dalan

Bailly, Benjamin

Bailly, Jean Luc

Balachandran, Siddharth

Balaraman, Velmurugan

Balasch, Monica

Balestrieri, Emanuela

Bálint, Ádám

Balkema-Buschmann, Anne

Balla, Keir M.

Ballegeer, Marlies

Bamford, Connor

Banadyga, Logan

Bańbura, Marcin W.

Bandea, Claudiu

Banerjee, Nilam Sanjib

Bánfalvi, Zsófia

Bao, Xiaoyong

Bara, Jeffrey

Barbeau, Benoit

Barbezange, Cyril

Barbic, Ljubo

Barbirz, Stefanie

Barjesteh, Neda

Barklis, Eric

Barr, Kelli L.

Barrett, Damien

Barril, Xavier

Barrs, Vanessa

Bartee, Eric

Bartholomeeusen, Dr. Koen

Bártolo, Inês

Bartowsky, Eveline

Barzon, Luisa

Battesti, Aurelia

Baur, Andreas

Bausch, Daniel

Baxter, Victoria K.

Bay, R Curtis

Baz, Mariana

Bażanów, Barbara

Beatty, Julia Anne

Beauvais, Wendy A.

Beaver, John R.

Becher, Paul

Becker, Stefanie Christine

Beckham, J. David 
Beckham, John David

Becking, Thomas

Beerens, Nancy

Behar, Adi

Behrens, Sven-Erik

Behzadi, Payam

Bejarano, Eduardo R.

Bejerman, Nicolas

Bell, Brendan

Belliot, Gaël

Bellizzi, Anna

Belogurov, Georgi

Belov, George

Belser, Jessica

Belsham, Graham

Belykh, Olga

Benaroch, Philippe

Bender, Scott C.

Benest, Andrew

Benfield, David A.

Bénichou, Serge

Benitez, Laura

Benítez, María José

Bennett, Antonette

Bennett, Richard S

Ben-Shachar, Rotem

Bergström, Tomas

Beringue, Vincent

Berkhout, Ben

Bernacchi, Serena

Bernatchez, Jean A.

Berrens, Rebecca

Bertho, Nicolas

Berthon, Patricia

Bertin, Sabrina

Bertoni, Giuseppe

Bertzbach, Luca Danilo

Best, Simon R.A.

Beyer, Wolfgang

Bhaskar, Sonu

Bhattacharjee, Apurba Krishna

$\mathrm{Bi}$, Xiuqiong

Bibby, David

Bickerton, Erica

Bicout, Dominique

Bideshi, Dennis

Biedenkopf, Nadine

Bielefeldt-Ohmann, Helle

Bienzle, Dorothee

Bierle, Craig

Biernat, Beata
Bigarré, Laurent

Biliavska, Liubov

Billerbeck, Eva

Binder, Marco

Bingham, John

Bisaillon, Martin

Bishop-Lilly, Kimberly A.

Bissonnette, Nathalie

Biswas, Santanu

Biswas-Fiss, Esther E.

Black, Lindsay

Blackstone, Neil

Blacquière, Tjeerd

Blahak, Silvia

Blair, Carol

Blair, Geoger Eric

Blaise-Boisseau, Sandra

Blanchard, Yannick

Blanco-Melo, Daniel

Blasdell, Kim

Blazquez, Ana-Belen

Blutt, Sarah Elizabeth

Bock, C. Thomas

Bodem, Jochen

Boehme, Amelia K.

Boete, Christophe

Bogers, Willy M J M

Boglione, Lucio

Boissot, Nathalie

Bojko, Jamie

Bollyky, Paul

Bolshoy, Alexander

Bonnefoy, Eliette

Bono, Hidemasa

Boon, Adrianus C. M.

Bopegamage, Shubhada

Borgmann, Kathleen

Born, Erwin Van Den

Borodovsky, Mark

Boroń-Kaczmarska, Anna

Borrego, Belén

Borsetti, Alessandra

Borucki, Monica

Bosco, Anthony

Bosco-Lauth, Angela M

Bosco-Lauth, Angela M.

Bošková, Veronika

Bosse, Jens B.

Bostina, Mihnea

Botella Sánchez, Leticia

Boudreau, Jeanette 
Boulanger, Pascale

Boulant, Steeve

Bouřa, Evžen

Bourgoin, Sandrine

Bourquain, Daniel

Bourret, Vincent

Bowen, Richard

Boyer, Thomas

Brackney, Douglas E.

Bradley, Todd

Brai, Annalaura

Brandt, Curtis

Brandt, Sabine

Brasier, Allan R.

Brenner, Bluma G.

Bressanelli, Stéphane

Breyta, Rachel

Breyton, Cécile

Brien, James

Brindley, Melinda

Brinkmann, Annika

Brinton, Margo

Brisse, Morgan

Brnić, Dragan

Brocato, Rebecca

Brochot, Etienne

Broecker, Felix

Brookes, Sharon

Brookes, Sharon M

Brown, Amanda M.

Brown, Ashley N.

Brown, Katherine

Brown, Paul A

Brown, Richard J.P.

Brown, Teagan

Browne, Edward P.

Bruce, Emily A.

Bruin, Erwin De

Brum, Jennifer R.

Brune, Wolfram

Brutscher, Laura

Bryan S., Kaplan

Bryant, Bart

Bryden, Steven

Brzozowska, Ewa

Buchanan, Tore

Buchko, Garry W.

Buck, Christopher B.

Buening, Hildegard

Bujarski, Józef J.

Bull, Jim
Burckhardt, Christoph

Burdo, Tricia H.

Burges, Graham

Burrone, Oscar Roberto

Burwitz, Benjamin J.

Buseyne, Florence

Buttimer, Colin

Byadgi, Omkar Vijay

Byrne, Alexander

Cabezon, Oscar

Cadar, Daniel

Caddy, Sarah

Cafiero, Mauricio

Cagliani, Rachele

Cagno, Valeria

Cai, Yingyun

Calderaro, Adriana

Calendar, Richard

Calistri, Arianna

Calistri, Paolo

Caller, Laura

Calvignac-Spencer, Sébastien

Calzolari, Mattia

Camarasa, María-José

Cambillau, Christian

Camero, Michele

Camp, Jeremy V.

Campbell, Corey L.

Campbell, Ellsworth

Campbell, Ellsworth M.

Campisciano, Giusi

Campos, Fabrício S.

Campos, Rafael Kroon

Camus-Bouclainville, Christelle

Candela, Monica G.

Cannon, Paula

Cantore, Alessio

Canuti, Marta

Cao, Dianjun

Cara, Andrea

Cardenas-Garcia, Stivalis

Cardeti, Giusy

Cardin, Rhonda

Careem, Faizal

Carey, Clayton

Carlton, Jeremy

Carmona-Vicente, Noelia

Carnaccini, Silvia

Carnahan, Robert H.

Caron, Alexandre

Carow, Berit 
Carpentier, David

Carr, Jillian M.

Carter, Carol A.

Caruso, Calogero

Caruso, Steven

Casartelli, Nicoletta

Casasnovas, Jose María

Case, James Brett

Casjens, Sherwood

Casola, Antonella

Castanha, Priscila M Da Silva

Castelletti, Noemi

Castillo, Daniel

Castle, Alan

Catalão, Maria João

Caughey, Byron

Cella, Eleonora

Cerny, Jan

Cetinel, Sibel

Chabannes, Matthieu

Chambers, Joseph E

Chambers, Thomas

Chandra, Naresh

Chandrashekar, Abishek

Chang, Chungjan

Chang, Hui-Wen

Chang, Jaewon

Chang, Pengxiang

Chang, Poa-Chun

Chang, Yen-Chen

Chao, Day-Yu

Chao, Mei

Chao, Yu-Chan

Chapman, Nora M.

Chapuy-Regaud, Sabine

Charleston, Michael

Charon, Justine

Charvat, Robert

Chase, Christopher

Chattopadhyay, Saurabh

Chatzopoulou, Fani

Chaurasiya, Shyambabu

Chauveau, Lise

Chavez-Calvillo, Gabriela

Chawla-Sarkar, Mamta

Chazal, Nathalie

Chebloune, Yahia

Chemin, Isabelle

Chen, Bing

Chen, Chunhong

Chen, Feng
Chen, Hui-Chen

Chen, $\mathrm{Li} \mathrm{Li}$

Chen, Shi-Jie

Chen, Wei-June

Chen, Yanping (Judy)

Chen, Yewang

Cheng, Haili

Cheng, Xiaoming

Cheng, Xiaowen

Cheng, Xiao-Wen

Cherepanov, Peter

Cheshenko, Natalia

Chesnais, Quentin

Che-Yen Wang, Joseph

Chiappinelli, Katherine

Chiapponi, Chiara

Chiba, Sotaro

Chibo, Doris

Chico Gras, Verónica

Chihade, Joe

Chikh Ali, Mohamad

Chinnakannan, Senthil

Chira, Sergiu

Chiu, Christopher

Chiumenti, Michela

Chlanda, Petr

Cho, Jae-Hyun

Cho, Nam-Hyuk

Cho, Tae-Ju

Cho, Won Kyong

Choi, Tae-Jin

Choi, Young-Ki

Chomont, Nicolas

Chow, Yen-Hung

Chowdhury, Ratul

Chowdhury, Shafiqul

Chowell, Gerardo

Christiaens, Olivier

Christian, Kimberly M

Christie, Gail

Chromy, David

Chua, Kaw Bing

Chulanov, Vladimir

Chulanov, Vladmir

Chung, Donghoon

Chung, Hui-Min

Ciccozzi, Massimo

Cilia, Giovanni

Cillo, Fabrizio

Cimica, Velasco

Cimolai, Nevio 
Cinek, Ondrej

Cingolani, Gino

Ciotti, Marco

Clarke, Penny

Claverie, Jean-Michel

Clement, Sophie

Cliffe, Anna

Clifford, David B.

Clouthier, Sharon

Cobbin, Joanna

Cobo, Jordi Serra

Cocklin, Simon

Coffin, John M.

Cohen, Daniel

Cohrs, Randall

Colavita, Francesca

Coller, Beth-ann

Collisson, Ellen

Colpitts, Che C.

Comas-Garcia, Andreu

Comas-Garcia, Mauricio

Combet, Christophe

Compeer, Ewoud B.

Compton, Alex

Conaldi, Pier Giulio

Confer, Anthony W.

Cong, Yingying

CongBao, Kang

Connerton, Ian

Coombs, Kevin

Cooper, Callum

Corcoran, Jennifer

Corcoran, Jennifer A

Cork, Susan

Correa, María

Cortese, Mirko

Cortez, Valerie

Corti, Manuela

Cortjens, Bart

Cory, Theodore J.

Cosby, Louise

Cosseddu, Gian Mario

Cosset, François-Loïc

Costa, Ana Rita

Costafreda Salvany, Maria Isabel

Costantini, Veronica P.

Costanzo, Michele

Côté, Marceline

Cotelle, Philippe

Cotten, Matthew

Couderc, Thérèse
Coulibaly, Fasseli

Cox, Andrea L.

Cox, Robert

Craig, Morgan

Crawford, Lindsey

Creager, Hannah

Crepin, Thibaut

Crisci, Elisa

Crocchiolo, Roberto

Croft, Larry

Crook, Brian

Csorba, Tibor

Cuesta, Alberto

Cui, Hongyu

Cullen, Bryan

Cun, Wei

Cusi, Maria Grazia

Cynis, Holger

Czosnek, Henryk

D'Angelo, Alberto

Da Costa, Rui M. Gil

Da Fonseca, Flavio Guimaraes

Dabrowska, Krystyna

Dacheux, Laurent

Dahle, Maria K

Dalal, Vijit

Dalgaard, Tina Sørensen

Dalianis, Tina

Dalmon, Anne

Daly, Janet

Damtie, Debasu

D'Andrea, Marco Maria

Darpel, Karin

Das, Kalyan

Dastjerdi, Akbar

Datta, Siddhartha

Daugrois, Jean-Heinrich

Davido, David

Davidson, Andrew

Davidson, Irit

Davies, Bryan William

Davis, April D.

Davis, David A.

Davydova, Julia

Dawson, Erica D.

De Alwis, Ruklanthi (Rukie)

De Benedictis, Paola

De Bernardi Schneider, Adriano

De Breyne, Sylvain

De Chiara, Giovanna

De Clercq, Erik 
De Francesco, Raffaele

De Gascun, Cillian F.

De Graaf, Miranda

De Groof, Ad

De La Pena, Marcos

de la Torre, Juan C.

De Marco, Maria Alessandra

De Miranda, Joachim R.

De Noronha, Carlos

De Paola, Domenico

De Regge, Nick

De Souza, William Marciel

De Swart, Rik

De Toni, Luca

De Vos, Daniel

De Vries, Rory D.

Deback, Claire

Debadyuti, Ghosh

Deboutte, Ward

Debyser, Zeger

DeCaprio, James

Decaro, Nicola

Decker, Aldo

DeFilippis, Victor R.

Deim, Zoltán

Del Fresno Sánchez, Carlos

Del Real, Gustavo

Del Valle, Luis

Delhon, Gustavo

Delhon, Gustavo A.

Della Bartola, Michele

Delputte, Peter

Delwart, Eric

Demangeat, Gérard

Dembowski, Jill

Deng, Xufang

Deniziak, Stanisław

Denzin, Nicolai

Depaquit, Jerome

Desbiez, Cécile

Desprès, Philippe

Desselberger, Ulrich

Destainville, Nicolas

DeStefano, Jeffery

Deutskens, Fabian

Devaux, Patricia

Deviatkin, Andrei A.

Dhakal, Santosh

Dhungel, Pragyesh

Di Bella, Santina

Di Gennaro, Francesco
Di Giallonardo, Francesca

Di Martino, Barbara

Di Micco, Simone

Di Nunzio, Francesca

Díaz Mochón, Juan José

Diaz, Arturo

Diaz-Griffero, Felipe

Diaz-San Segundo, Fayna

Dick, Robert

Diederich, Sandra

Dieterle, María Eugenia

Dietrich, Isabelle

Dietrich, Ursula

Dietzgen, Ralf

Dijkman, Ronald

Dikeakos, Jimmy

Dimienescu, Oana Gabriela

Dimitrov, Kiril

Dimitrov, Kiril M.

Dineley, Kelly

Ding, Shou-Wei

Diskin, Ron

Dobbler, Gerhard

Dodds, W. Jean

Dokland, Terje

Dolei, Antonina

Domanovic, Dragoslav

Domańska-Blicharz, Katarzyna

Dombrovsky, Aviv

Domenech, Ana

Domier, Leslie

Domingo, Cristina

Domingo, Mariano

Domingo-Calap, Pilar

Donadu, Matthew

Donaire, Livia

Donald, Claire L.

Donato, Celeste

Dong, Shengzhang

Doore, Sarah M.

Dopazo, Carlos P.

Dorotkiewicz-Jach, Agata

D'orso, Ivan

Dosoky, Noura

Doszpoly, Andor

Doty, Jeffrey

Dou, Xiaoguang

Doublet, Vincent

Douglas, Mark W.

Dowd, Kimberly A.

Dražić, Maja 
Drebot, Mike

Drigo, Michele

Drillien, Robert

Drolet, Barbara

Drozd, Radoslaw

Druce, Julian

Drucker, Martin

Drummer, Heidi

$\mathrm{Du}$, Juan

$\mathrm{Du}, \mathrm{Ke}$

Dubé, Mathieu

Dubovi, Edward

Ducatez, Mariette

Ducatez, Mariette F.

Duchêne, Sebastián

Dudley, Jaquelin P.

Dudman, Susanne G.

Dugdale, Benjamin

Duke, Elizabeth

Dunne, Matthew

Dunowska, Magdalena

Durand, Guillaume A.

Dürrwald, Ralf

Dutartre, Hélène

Dutch, Rebecca

Dvorak, Cheryl

Dyall, Julie

Dyall-Smith, Mike

Dykeman, Eric

Dziewit, Lukasz

Eastwell, Kenneth C.

Ebert, Greg

Ebrahimi, Diako

Echevarría, Juan E.

Ecke, Thorsten

Eckels, Kenneth H.

Eden, John-Sebastian

Edenborough, Kathryn

Edgar, James

Edlefsen, Paul T.

Egberink, Herman

Eggers, Christian $\mathrm{H}$

Eggink, Dirk

Egyed, László

Ehlers, Bernhard

Ehmann, Rosina

Ehrhardt, Anja

Ehrlich, Marcelo

Eichwald, Catherine

Elbadry, Maha A

Eldar, Avi
Elena, Criscuolo

El-Gazzar, Mohamed M.

Elghobashi-Meinhardt, Nadia

El-Hage, Nazira

Elliman, Jennifer

El-Mayet, Fouad

Elofsson, Mikael

Elsayed, Ahmed Mostafa

Elshabrawy, Hatem A.

Elshesheny, Rabeh

Emam, Mehdi

Emerson, Joanna

Endo, Hisashi

Engeland, Christine E.

England, Marion

Enjuanes, Luis

Enosi Tuipulotu, Daniel

Ergunay, Koray

Eriko, Ohsaki

Ermonval, Myriam

Ertl, Reinhard

Eschbaumer, Michael

Escribano-Romero, Estela

Escriu, Fernando

Escudero-Abarca, Blanca

Escudero-Pérez, Beatriz

Eskelin, Katri

Essani, Karim

Etebari, Kayvan

Etienne, Lucie

Ettayebi, Khalil

Evani, Shankar Jaikishan

Everett, Helen E.

Evermann, Jim

Ewans, Matthew

Ewing, Adam

Eyre, Nicholas

Faaberg, Kay

Fabeni, Lavinia

Fablet, Christelle

Faccini, Silvia

Faita, Francesco

Falendysz, Elisabeth A.

Falkenberg, Shollie

Falkenberg, Shollie M.

Fan, Wenchun

Fan, Yi-Chin

Farenc, Carine

Farkas, Silvie

Fast, Mark

Faulk, Christopher D. 
Faulkner, Geoffrey John

Fauver, Joseph

Favia, Guido

Favier, Anne-Laure

Fehr, Anthony

Feiss, Michael G.

Feliziani, Francesco

Feng, Cong

Feng, Ningguo

Fera, Daniela

Ferdinandy, Bence

Fereres, Alberto

Ferguson, Brian

Fernández, Lucía

Fernandez, Melissa

Fernández-Alarcón, Claudia

Ferrara, Francesca

Ferreira, Fernando

Ferrer, Isidro

Ferrero, Diego

Ferrés, Marcela

Ferriol, Inmaculada

Ferron, François

Ferry, Tristan

Feschotte, Cédric

Fibriansah, Guntur

Fichet-Calvet, Elisabeth

Figuerola, Jordi

Filipič, Bratko

Finetti-Sialer, Mariella M.

Finlaison, Deborah S.

Fiorentini, Simona

Fischer, Carlo

Fischer, Melina

Fischer, Nicole

Fiume, Giuseppe

Flannery, John

Flehmig, Bertram

Fleming, Damarius

Flenniken, Michelle

Flenniken, Michelle L.

Fleury, Hervé

Focosi, Daniele

Folegatti, Pedro

Foley, Brian T.

Fonseca, Gregory J.

Fonseca, Kevin

Fontana, Juan

Fontanay, Stéphane

Foppa, Ivo M.

Ford, Kathryn
Forni, Diego

Forrest, Craig

Forrester, Naomi

Forsman, Mats

Forstova, Jitka

Fortuna, Claudia

Forzan, Mario

Fossé, Philippe

Fossen, Torgils

Foster, Toshana Lauria

Fox, Julie

Foxi, Cipriano

Frada, Miguel

Fralick, Joe A.

França, Monique S.

Francis, Ashwanth Christopher

Franco, David

Francois, Catherine

François, Sarah

Frangeul, Lionel

Frank, Luiza

Frant, Maciej

Franzoni, Giulia

Fraune, Sebastian

Freiberg, Alexander N.

Freimanis, Graham

Frentiu, Francesca

Frese, Michael

Freuling, Conrad

Frias Casas, Mario

Froissart, Rémy

Frolova, Elena

Fros, Jelke

Frossard, Jean-Pierre

Frunzke, Julia

Frutos, Roger

$\mathrm{Fu}$, Tong-Ming

Fuchs, Marc

Fuji, Shin-Ichi

Fujikura, Daisuke

Fujita, Ryosuke

Fujiwara, Kei

Fukuhara, Takasuke

Fukushi, Hideto

Fukuta, Shiro

Fukuzawa, Noriho H.

Fuller, Frederick J.

Fuller, Trevon

Furlini, Giuliano

Furuse, Yuki

Furuya, Tetsuya 
Furuya, Yoichi

Fusco, Dahlene

Gabriel, Emmanuel

Gaffuri, Alessandra

Gaglia, Marta Maria

Gagne, Roderick

Gajda, Anna

Galabov, Angel

Gałas, Aleksander

Gale, Patrick

Gallagher, Thomas

Gallardo, Rodrigo

Gambardella, Claudio

Gambarian, Alexandra

Gambino, Giorgio

Gammon, Don

Ganaie, Safder

Ganges, Llilianne

Ganji, Rakesh

Gann, Eric R.

Ganor, Yonatan

Ganser-Pornillos, Barbie

Ganser-Pornillos, Barbie K.

Gao, Yong

Garbuglia, Anna Rosa

García, Pedro

Garcia-Dorival, Isabel

Garcia-Estañ, Luis Perez

García-Murria, María J.

Garcia-Ruiz, Hernan

Gardner, Matthew J.

Garfinkel, David

Garigliany, Mutien-Marie

Garofalo, Mariangela

Garten, Wolfgang

Gascuel, Olivier

Gastaminza, Pablo

Gattinger, Pia

Gaudin, Yves

Gaudreault, Natasha N

Gauger, Phillip C.

Gehrke, Lee

Geisler, Christoph

Geldenhuys, Marike

Gendrot, Mathieu

Generoso, Jaqueline Da Silva

Geng, Tingting

Gerber, Priscilla F.

Gerhauser, Ingo

Gerlich, Wolfram

Gershon, Michael
Ghosh, Souvik

Ghosh, Sumit

Giadinis, Nektarios D.

Giammanco, Giovanni M

Giangaspero, Massimo

Giannecchini, Simone

Gifford, Robert

Gil, Jose Fernando

Gill, Jason

Gimenez-Lirola, Luis G.

Ginet, Nicolas

Giovanardi, Davide

Gish, Robert

Giuffrida, Paolo

Gladue, Douglas

Glasa, Miroslav

Glenn, Wendy

Glowacz, Adam

Goffinet, Christine

Goh, Gerard Kian-Meng

Goldbourt, Amir

Goldstein, Richard

Goldstein, Ronald

Golec, Piotr

Golender, Natalia

Golender, Natalya

Goletti, Delia

Golubchik, Tanya

Golubovskaya, Vita

Gómez Castilla, Jordi

Gomez Monterrey, Isabel

Gomez-Lucia, Esperanza

Gong, Bin

Gong, Peng

Gonzales, Jose L

Gonzalez, Gabriel

Gonzalez, Jean-Paul

Gonzalez, John-Paul

González-Bulnes, Antonio

Gonzalez-Dunia, Daniel

Gonzalez-Reiche, Ana Silvia

Goodier, Martin R.

Goodman, Alan G.

Goodman, Cynthia L.

Goodwin, Thomas

Gossner, Céline Marie Elise

Gouandjika-Vasilache, Ionela

Gozalbo Rovira, Roberto Vicente

Gralinski, Lisa

Grand, Roger

Grandi, Nicole 
Grant, Michael

Green, Kim

Green, Todd

Greiner, Timo

Greive, Sandra

Griffin, Heather

Griffiths, David J.

Grimsley, Nigel

Grose, Julianne

Grose, Julianne H.

Grove, Joe

Groves, Ian

Grubman, Marvin

Grünweller, Arnold

$\mathrm{Gu}$, Zhen

Guardado-Calvo, Pablo

Guerra-Assuncao, Afonso

Guerrero-Plata, Antonieta

Guerrini, Marco

Guido, Poli

Guillaume, Mousseau

Guillon, Christophe

Guo, Ming-Lei

Guo, Zong Sheng

Gupta, Kapil

Gustin, Kurt

Gutiérrez-López, Rafael

Guy, Bruno

Guy, Paul L.

Guydosh, Nicholas R.

Győző, Kaján

Gysi, Deisy

Gyula, Péter

Haan, Peter De

Haase, Astrid D.

Habili, Nuredin

Hadidid, Ahmed

Hadziyannis, Emilia

Haese, Nicole N.

Hagiwara, Katsuro

Hahm, Bumsuk

Hahn, Alexander

Hai, Rong

Hairgrove, Thomas

Hajarizadeh, Behzad

Hakami, Ramin

Hakata, Yoshiyuki

Haley, Sheila A.

Hall, Roy A.

Hallden, Gunnel

Hall-Mendelin, Sonja
Hamel, Rodolphe

Hammarskjöld, Marie-Louise

Hammond, John

Hampson, Lynne

Han, Hui-Ju

Han, Lei

Hang, Jun

Hankaniemi, Minna M.

Hanks, Ephraim M.

Hann, Hie-Won

Hans, Aymeric

Haqshenas, Gholamreza

Haque, Muzammel

Harder, Timm

Hardies, Stephen C.

Harding, Rob

Harmon, Karen M.

Harper, David R.

Harrach, Balázs

Harrich, David

Harris, Audray

Harris, Reuben

Harrison, Robert

Harrod, Robert

Hartert, Tina V.

Hartshorn, Kevan L

Hartung, Hans-Peter

Harty, Ronald

Harvey, William

Hassan, Sharifah Syed

Hatano, Yuichiro

Hatfull, Graham

Hattori, Toshio

Hause, Ben

Hawes, Philippa

Hay, Iain D

Hayasaka, Daisuke

Hayer, Juliette

Hayes, Sidney J.

Hazan, Ronen

He, Jie

He, Mike Z.

He, Qigai

Hearing, Patrick

Hébrard, Eugénie

Hedman, Klaus

Hefferon, Kathleen L.

Hefferon, Kathleen Laura

Hegele, Richard G

Heinlein, Manfred

Heitz, Thierry 
Hejnar, Jiri

Helbig, Karla

Helle, Francois

Henderson, Andrew

Henderson, Andrew J.

Heng, Xiao

Henke, Andreas

Henrich, Curtis J.

Hepojoki, Jussi

Herchenröder, Ottmar

Herker, Eva

Hernáez, Bruno

Hernández, Carmen

Hernandez, Jesus

Hernandez-Vargas, Esteban

Hernandez-Vargas, Esteban A.

Herod, Morgan

Herrera-Carrillo, Elena

Hersperger, Adam R.

Hertel, Robert

Herzog, Catherine

Heselpoth, Ryan

Hesson, Jenny

Hicks, Julie A.

Hijano, Diego R.

Hildt, Eberhard

Hill, Fraser

Hillyer, Julian

Hily, Jean-Michel

Hingamp, Pascal M.

Hinkula, Jorma

Hinton, Deborah

Hipp, Katharina

Hirayama, Kazuhiro

Hirono, Ikuo

Hirota, Jeremy

Hirsch, Alec J.

Hirsch, Hans

Hirsch, Ivan

Hirsch, Judith

Hirsch, Silvia Ayora

Hizi, Amnon

Ho, Chak-Sum

Ho, Eric S.

Ho, Ya-Chi

Hobson-Peters, Jody

Hodcroft, Emma B.

Hoeben, Rob

Hoek, Lia Van Der

Hoenen, Thomas

Hoffmann, Markus
Höfle, Ursula

Hofstetter, Amelia R.

Hogan, Michael J.

Hogue, Ian

Holbrook, Michael

Holicki, Cora M.

Hollister, Emily B.

Holmes, Edward C.

Hölzer, Martin

Holzhauer, Menno

Homa, Fred L.

Hong, Xupeng

Hopken, Matthew W.

Horhat, Florin George

Horie, Masayuki

Horimoto, Taisuke

Horton, Nancy

Horton, Nancy C.

Horwood, Paul F

Hosmalin, Anne

Hostnik, Peter

Hou, Yixuan

Houldcroft, Charlotte

Houston, Fiona

Hovi, Tapani

Hraber, Peter

Hribar, Lawrence

Hristov, Peter Ivanov

Hsieh, Ming Kun

Hsieh, Shie-Liang

$\mathrm{Hu}$, Jiafen

$\mathrm{Hu}$, Yanmei

Huang, Cheng

Huang, Chienjin

Huang, Hsing I

Huang, Jason C.

Huang, Yong

Huang, Ziwei

Hubai, András

Huber, Andrew

Huber, Victor

Hufbauer, Martin

Hufton, Simon E.

Hughes, Holly R.

Hughes, Joseph

Hughes, Stephen

Hume, Adam

Hummel, Mary

Hurst, Christon

Hutson, Christina

Hwang, Eung-Soo 
Hynes, Alexander

Hyodo, Kiwamu

Hytönen, Vesa

Ibrahim, Ahmed

Ikebuchi, Ryoyo

Ikeda, Terumasa

Imai, Masaki

Imbeault, Michael

Incarnato, Danny

Indugu, Nagaraju

Inoue, Jun

Iordanskiy, Sergey

Iorio, Ronald M.

Isakova-Sivak, Irina

Isel, Catherine

Iseni, Frédéric

Ishibashi, Kazuhiro

Ishikawa, Masayuki

Ishikawa, Tomohiro

Ishima, Rieko

Ishizaki, Azumi

Iskra-Caruana, Marie-Line

Islam, Ariful

Ito, Kimihito

Ito, Naoto

Iturriza-Gomara, Miren

Ivankovic, Tomislav

Iwano, Hidetomo

Iwanowicz, Luke R

Iwasaki, Masaharu

Izsvák, Zsuzsanna

J. Jackwood, Daral

Jääskeläinen, Anne J.

Jackova, Anna

Jackson, Brian

Jackson, Kathy M.

Jackson, Sarah E.

Jacob, Gopas

Jacobs-Sera, Deborah

Jadhao, Samadhan J.

Jaguva Vasudevan, Ananda Ayyappan

Jaimes, Javier A.

Jakab, Ferenc

Jakimovski, Dejan

Jakobsson, Johan

Jalava, Katri

James, Burke

James, Claire D

James, William

Jamin, Marc

Jan, Eric
Jan, Fuh-Jyh

Jancovich, James

Janelle, Valerie

Jangra, Rohit

Janowski, Andrew B.

Jansen, C.A. (Christine)

Jansen, Stephanie

Jardine, Paul

Jarocki, Piotr

Jarosinski, Keith

Jeger, Michael

Jenckel, Maria

Jenkins, Frank

Jeonga, Dae Gwin

Jeske, Holger

Jiang, Guochun

Jiang, Qiu-Xing

Jiang, Shijin

Jiang, Sizun

Jiménez De Oya, Nereida

Jin, Dong-Yan

Jin, Jing

Jin, Tengchuan

Jin, Yuefei

Jochmans, Dirk

Jõers, Priit

John, Sinu P.

Johnson, John

Johnson, Nicholas

Johnson, Reed F.

Johnston, J. Spencer

Johnston, Randal N.

Jończyk-Matysiak, Ewa

Jones, Bryony A.

Jones, Clinton

Jones, Ian

Jones, Jeremy

Jones, Melissa

Jordan, Brian

Jordan, Jeanne A.

Jordan, King

Jordan, Michael R

Jørgensen, Charlotte Sværke

Jose, Joyce

Joshi, Hiren

Josset, Laurence

Julander, Justin G.

Jung, Alain

Jung, Yong Sam

Jurak, Igor

Jurvansuu, Jaana 
Kaczmarek, Maria E.

Kaczorowski, Tadeusz

Kading, Rebekah C.

Kaelber, Jason

Kafri, Tal

Kageyama, Tsutomu

Kainulainen, Markus H

Kaján, Győző L.

Kajihara, Masahiro

Kalinowski, Jörn

Kallies, Rene

Kamau, Everlyn

Kanda, Tatsuo

Kanda, Teru

Kandeel, Mahmoud

Kang, Sang-Moo

Kant, Sashi

Kantor, Asher

Kaplan, Shauna

Karimi, Khalil

Karlsson, Erik

Karniychuk, Uladzimir

Károly, Fátyol

Karsten, Christina Beatrice

Karuppannan, Anbu Kumar

Kashanchi, Fatah

Kashiwagi, Akiko

Kathuria, Himanshu

Katneni, Upendra

Katoh, Hiroshi

Katsoulos, Panagiotis D.

Katz, Richard

Katzenellenbogen, Rachel A.

Kaufer, Benedikt B.

Kaufmann, Andreas

Kaul, Marcus

Kautz, Tiffany F.

Kawada, Jun-ichi

Kay, Brian

Kayali, Ghazi

Kazama, Shinobu

Ke, Po-Yuan

Kearney, Mary F.

Kedzierska, Katherine

Kehn-Hall, Kylene

Keita, Alpha

Kelch, Brian

Kell, Alison

Kelly, Gabrielle

Kemenesi, Gábor

Kennedy, Melissa
Kennedy, Richard B.

Kenney, Joan L.

Kenney, Scott P.

Khaperskyy, Denys A.

Khatri, Vishal

Khayat, Reza

Kheimar, Ahmed

Khurshid, Zohaib

Kibenge, Frederick

Kibler, Karen V.

Kiefer, Dorothee

Kiełpińska, Jolanta

Kieran, Troy J

Kierzek, Elzbieta

Kiga, Kotaro

Kil, Eui-Joon

Kile, James C.

Killian, Mary Lea

Kilpatrick, Marm

Kim, Chonsaeng

Kim, Dal Young

Kim, Hye Kwon

Kim, In-Jeong

Kim, Jee Hyun

Kim, Joomyeong

Kim, Ki-Hong

Kim, Meehyein

Kim, Mikyeong

Kim, Seil

Kim, Su-mi

Kimpel, Janine

Kimura, Hirokazu

Kincaid, Rodney

Kinchington, Paul

King, Donald

King, Linda

Kingsley, David

Kipar, Anja

Kirchhoff, Frank

Kireev, Dmitry

Kirkwood, Carl

Kirubakaran, Palani

Kirui, James

Kis, Zoltan

Kiseleva, Irina V.

Kishida, Yutaka

Kiso, Maki

Kitchen, Andrew

Klapper, Paul

Klase, Zachary A.

Klein, Terry A. 
Kleinow, Tatjana

Kleinpeter, Alex

Klenk, Hans-Dieter

Klimkait, Thomas

Klonjkowski, Bernard

Klose, Thomas

Klyczek, Karen

Knepper, Janice

Knight, Richard J.

Knowles, Graeme

Knowles, Nick J.

Ko, Eun-ju

Kobayashi, Tetsuro

Kobie, James J.

Kobiler, Oren

Kochneva, Galina

Kock, Richard

Kodidela, Sunitha

Koethe, Susanne

Kohara, Michinori

Koivisto, Janne

Kolawole, Abimbola

Kolbasov, Denis

Kolishetti, Nagesh

Komar, Nicholas

Komatsu, Ken

Komorowska, Beata

Kondili, Loreta

Kondo, Hideki

Kondo, Satoru

König, Alexander

Konjevic, Dean

Koodie, Lisa

Koos, Björn

Kordyukova, Larisa

Kormelink, Richard

Kormuth, Karen

Koromyslova, Anna

Korth, Johannes

Koshiba, Takumi

Koshizuka, Tetsuo

Kosik, Ivan

Koskan, Alexis

Koslová, Anna

Kostaki, Evangelia

Kostyushev, Dmitry

Kosulin, Karin

Kotta-Loizou, Ioly

Kouyos, Roger D

Kovarik, Ales

Kozak, Christine
Kozieł, Edmund

Koziol-White, Cynthia J.

Kozlovskaya, Liubov

Kozlowski, Lukasz

Krammer, Florian

Kraska, Thorsten

Krejbich-Trotot, Pascale

Krenz, Björn

Krishnakumar, Devadas

Krishnamurthy, Siddharth R.

Kroeker, Andrea

Krol, Ewelina

Krug, Laurie

Krug, Peter

Kruger, Detlev

Krüger, Nadine

Kruse, Robert L.

Kryger, Per

Krylov, Victor

Krzyzowska, Malgorzata

Kubo, Yoshinao

Kucheryavykh, Lilia Y.

Kugelman, Jeffrey R.

Kuhar, Urška

Kula-Pacurar, Anna

Kulkarni, Amol

Kumar Kundu, Jiban

Kumar, Amit

Kumar, Ashok

Kumar, Asit

Kumar, Binod

Kumar, Mukesh

Kumar, Penmetcha

Kumar, Shailesh

Kumari, Asha

Kümmerer, Beate M.

Kunec, Dusan

Kuniya, Toshikazu

Kuo, Yen-Wen

Kurebayashi, Yuuki

Kurian, Justin J.

Kurt, Tobler

Küry, Patrick

Kuryk, Lukasz

Kusejko, Katharina

Kuss-Duerkop, Sharon K.

Kuzmak, Jacek

Kuzmin, Ivan

Kuzmina, Natalia A.

Kvale, Dag

Kwiatek, Olivier 
Kwilas, Steven

Kwon, Amy

Kwon, Sunoh

Kwun, Hyun Jin

Kyei, George B

L. Schmaljohn, Alan

La Rosa, Giuseppina

Laanto, Elina

Labo, Nazzarena

Lacey, Charles J. N.

Lagatolla, Cristina

Lagaudrière-Gesbert, Cécile

Lagunoff, Michael

Lai, Michael M. C.

Laksono, Brigitta

Lamb, Laura

Lamba, Doriano

LaMere, Sarah

Lanave, Gianvito

Lang, Andrew

Lang, Fengchao

Lange, Ulrike

Langel, Stephanie Neal

Langsjoen, Rose

Lanteri, Giovanni

Lattorff, H. Michael G.

Lauzi, Stefania

Laval, Kathlyn

Lawrence, Charles M.

Lazar, Catalin

Le Goffic, Ronan

Le Mercier, Philippe

Le Pendu, Jacques

Le Sage, Valerie

Leal, Ana Mendes

Lean, Fabian Z. X.

Lebrand, Aitana

Lednicky, John

Lee, Joo-Youn

Lee, Kelly

Lee, Keun Hwa

Lee, Kuo Hao

Lee, Min Young

Lee, Tzong-Shyuan

Leeks, Asher

Leeuw, Erik De

Lefkowitz, Elliot J.

Legendre, Mathieu

Legler, Patricia

Lehman, Dara A.

Lehman, John M.
Leifels, Mats

Leis, Jonathan P.

Leisi, Remo

Leitão, Alexandre

Lemay, Guy

Lemmermann, Niels A W

Lenz, Ondřej

Leon-Juarez, Moises

Lequime, Sebastian

Leroux, Caroline

Lesbats, Paul

Lester, Philip John

Letarov, Andrey

Letoha, Tamás

Leung, Anthony

Leung, Daisy W.

Leung, Kwong-Sak

Leutenegger, Christian M.

Levin, Judith

Levrero, Massimo

Levy, Ronald M.

Lewitus, Eric

Leymarie, Olivier

Lhomme, Sébastien

Li, Chunfeng

Li, Dapeng

Li, Hai

$\mathrm{Li}$, Hongmin

$\mathrm{Li}$, Jinquan

Li, Jonathan

$\mathrm{Li}$, Lin

Li, Melody Hing

Li, Renfeng

Li, Shitao

Li, Xiangmin

Li, Xiaojun

Li, Yanli

$\mathrm{Li}$, Zhuoran

Liang, Chen

Liang, Po-Huang

Liang, Yuying

Liao, Jiawen

Liao, Laura E.

Liao, Yu-Chieh

Liberski, Paweł P.

Lichterfeld, Mathias

Lim, Chun Shen

Lim, Siew Pheng

Lim, Stephanie M

Lin, Chao-Nan

Lin, Cheng-Wen 
Lin, Tao

Lindberg, A. Michael

Lindemann, Dirk

Lindenbach, Brett

Ling, Chen

Ling, Jiaxin

Ling, Jiqiang

Lingwood, Daniel

Linial, Maxine

Linial, Maxine L.

Lipińska, Andrea

Littlejohn, Margaret

Liu, GuanQun

Liu, Haolin

Liu, Hsin-Fu

Liu, Jia

Liu, Qiang

Liu, Shan-Lu

Liu, Wei

Liu, Xiaoying

Liu, Yue

Liu, Zhuoming

Lizano, Marcela

Lizarazo, Erley F.

Llanes, Alejandro

Llorente, Francisco

Lo Giudice, Roberto

Lo, Shih-Yen

Lo, Szecheng

Loc-Carrillo, Catherine

Lodmell, J. Stephen

Logue, James

Lohmann, Volker

Lojkić, Ivana

London, Robert E.

Londono, Berlin

Londrigan, Sarah

López, Carolina B.

Lopez-Ferber, Miguel

López-Ferber, Miguel

Lorenzo, Gema

Lorusso, Alessio

Los, Marcin

Lotz, Jeffrey

Louis, Irina St.

Lovell, Scott

Lowe, David

Lozach, Pierre-Yves

Lozano, Jose Manuel

Lozano-Durán, Rosa

Lu, Tzupin
Lucifora, Julie

Lühken, Renke

Lukacher, Aron

Lukashevich, Igor

Lukhovitskaya, Nina

Lulla, Valeria

Lundgren, Magnus

Lundstrom, Kenneth

Luo, Zhen

Lupberger, Joachim

Lüscher, Bernhard

Lustig, Yaniv

Luthra, Priya

Luttermann, Christine

Ly, Hinh

Lycett, Samantha

Lynch, Rebecca

Lynch, Stacey E

Lyu, Yuan

Ma, Wenjun

Mabashi-Asazuma, Hideaki

Macchi, Beatrice

Macdonald, Andrew

MacDuff, Donna A.

Macfarlan, Todd

Machado, Alexandre

Maciejewska, Barbara

Maciver, Sutherland

MacNeill, Amy

Maeda, Kazuhiko

Maeda, Naoyoshi

Maekawa, Shun

Mager, Dixie L.

Maggi, Fabrizio

Maggirwar, Sanjay

Maghsoodi, Ameneh

Maginnis, Melissa

Magor, Katharine

Mahar, Jackie

Mahony, Timothy John

Maillard, Pierre V

Maisetta, Giuseppantonio

Maixner, Michael

Majerciak, Vladimir

Majewski, Jacek

Majkowska-Skrobek, Grażyna

Mak, Lung-Yi

Makarova, Kira S

Makowski, Lee

Malagon, Francisco

Maldarelli, Frank 
Maldonado, Juan

Mamede, Joao

Mang, Stefania Mirela

Mangala Prasad, Vidya

Mangino, Giorgio

Manicassamy, Balaji

Mans, Janet

Manuel Giorgi, Federico

Manzoor, Rashid

Marc, Daniel

Marcacci, Maurilia

Marchant, David

Marchetti, Monia

Marchini, Antonio

Marchiò, Serena

Marenzoni, Maria Luisa

Margina, Denisa

Margulies, Barry

Marin Lopez, Alejandro

Mariner, Jeffrey

Maringer, Kevin

Marjomaki, Varpu

Mark S., Johnson

Markovich, Michal Perry

Markussen, Turhan

Marquet, Roland

Marriott, Anthony

Marschall, Manfred

Marsden, Matthew

Marston, Denise

Marszałek, Jarosław

Martella, Vito

Martikainen, Miika

Martín Castillo, Margarita

Martin, Annette

Martin, Eileen

Martin, Javier

Martin, Stephen

Martin, Tammy M.

Martín-Acebes, Miguel A.

Martinet, Jean-Philippe

Martinez, Jose C

Martínez, Miguel A.

Martínez-García, Manuel

Martinez-Gil, Luis

Martínez-Martínez, Irene

Martins, Nelson

Marty, Gary

Marutescu, Luminita

Marutescu, Luminita Gabriela

Maruyama, Junki
Marzano, Shin-Yi

Mas, Antonio

Masalova, Olga V.

Masataka, Tsuge

Massanella, Marta

Mastino, Antonio

Masuda, Takao

Matalka, Khalid Z.

Matczuk, Anna Karolina

Mateo, Mathieu

Mathew, Anuja

Mathieu, Cyrille

Mathijs, Elisabeth

Matsuo, Eiko

Matsushita, Michiko

Mattapallil, Joseph

Matthews, Louise

Matusali, Giulia

Mauffret, Olivier

Mavian, Carla

May, Jared

Mazurov, Dmitriy V

Mazzei, Maurizio

Mbonye, Uri

McBride, John

McCauley, Micah

McCormick, Craig

McCulloch, Karen

McDonnel, Samantha J.

McFadden, Grant

McFarlane, Donald A.

Mcllroy, Dorian

McInerney, Gerald

McKee, Clifton

Mcknight, Aine

McLean, Gary

McLean, Rebecca

McMillan, Nigel

McVey, Colin

Medina Piles, Vicente

Medina, Gisselle N.

Medina-Kauwe, Lali K.

Mediouni, Sonia

Mee, Edward T.

Meertens, Laurent

Mehedi, Masfique

Mehta, Sanjay R.

Mei, Ya-Fang

Meier-Stephenson, Vanessa

Melcher, Ulrich

Melikian, Gregory 
Meliopoulos, Victoria A

Melzer, Michael J.

Mena, Ignacio

Mencia Caballero, Mario

Mendoza, Emelissa

Menéndez-Arias, Luis

Meng, Xiangchao

Menne, Stephan

Menzo, Stefano

Mergia, Ayalew

Merino, Jose Joaquin

Merkling, Sarah Helene

Mesci, Pinar

Meškys, Rolandas

Mesri, Enrique

Metifiot, Mathieu

Metz, Stefan

Metz, Stefan W.

Metzger, Michael

Metzner, Karin

Meurens, Francois

Meurs, Eliane

Meuti, Megan

Meyer, Florencia

Meyer, Gilles

Meyer, Hermann

Meyer, Justin

Meyers, Craig

Miao, Qiuhong

Michaux, J. R.

Michon, Thierry

Miesen, Pascal

Milani, Adelaide

Milani, Mario

Milavetz, Barry

Millard, Andrew

Millard, Andrew D.

Miller, W. Allen

Miller-Saunders, Kristi

Millet, Jean K.

Minematsu, Toshio

Mirabelli, Carmen

Miranda, Carla

Miroslaw, Snietura

Mishra, Awdhesh

Mitsuru, Okuwaki

Miyashita, Shuhei

Miyazawa, Masaaki

Mizukami, Shusaku

Mlera, Luwanika

Mocarski, Edward
Mochizuki, Tomofumi

Modahl, Cassandra

Modha, Sejal

Moens, Ugo Lionel

Mohanty, Smita

Möhl, Britta

Mohr, Emma L.

Mohsen, Mona

Mok, Chris C.P.

Molineux, Ian

Monguió-Tortajada, Marta

Monne, Isabella

Montagutełli, Xavier

Montassier, Helio J.

Montes, Nuria

Montoya, Maria

Moore, Nicholas M.

Moore, Patrick

Morabito, Kaitlyn M.

Moraes, Theo

Morais, Marc

Moraru, Liliana Cristina

Mordecai, Gideon

Moreno, Miguel

Morgan, Ethan

Morgan, Ethan L.

Mori, Yasuko

Morimoto, Kinjiro

Morita, Eiji

Morley, Valerie

Morozov, Igor

Morozova, Olga

Morozova, Vera V.

Morris, Mhairi

Morrison, Juliet

Morrison, Thomas E. "Tem"

Moss, Bernard

Mossel, Eric

Mostafa, Ahmed

Mousa, Jarrod J.

Moutailler, Sara

Moutelíková, Romana

Mucker, Eric M.

Mühlberger, Elke

Mukhopadhyay, Tuli

Mulder, Lubbertus

Mulkey, Sarah

Mullarkey, Caitlin

Müller, Barbara

Muller, David Alexander

Müller, Janis 
Muller, Mandy

Munk, Carsten

Muñoz-Barroso, Isabel

Muntean, Alexandru Andrei

Murakami, Hironobu

Murakami, Kosuke

Murakami, Shin

Murakami, Yoshiki

Muramatsu, Masamichi

Murata, Takayuki

Muriaux, Delphine

Murillo, Rosa

Murota, Katsunori

Murovska, Modra

Murovska, Modra F.

Murphy, Alex M

Murphy, Eain

Murray, Shannon M.

Musalgaonkar, Sharmishtha

Mustafa, Ghazala

Muthu Karuppan, Mohan Kumar

Mutoloki, Stephen

Muxel, Sandra Marcia

Myers, Dean

Myung, Heejoon

Nachbagauer, Raffael

Naesens, Lieve

Nagafuchi, Seiho

Nagai, Makoto

Nagasaka, Kazunori

Naghavi, Mojgan H.

Naguib, Mahmoud

Naik, Shruthi

Naji, Hassan S.

Nakagawa, Koji

Nakagawa, So

Nakahara, Kenji S.

Nakahara, Tomomi

Nakakita, Shin-ichi

Nakanishi, Akira

Nakauchi, Mina

Nakayama, Eri

Nakayashiki, Hitoshi

Nakazawa, Yoshinori

Nanbo, Asuka

Nasar, Farooq

Navarro, Beatriz

Navas-Martin, Sonia

Navratil, Vincent

Nawrocki, Steffan T.

Nawrot, Robert
Nayak, Tapan

Nazli, Aisha

Neerukonda, Sabarinath

Nejman-Faleńczyk, Bożena

Nekhai, Sergei

Nelson, Scott W.

Nemes, Katalin

Nerva, Luca

Netherton, Christopher L.

Neufeldt, Christopher John

Neuman, Benjamin

Neumann, Donna

Nevola, Riccardo

Nice, Timothy

Nicoletti, Loredana

Niedźwiedzka-Rystwej, Paulina

Nielsen, Tue Kjærgaard

Niepmann, Michael

Niespodziana, Katarzyna

Nigam, Deepti

Niikura, Masahiro

Nishigaki, Kazuo

Nishihara, Hidenori

Nishizono, Akira

Nisole, Sébastien

Nissimov, Jozef

Nistal-Villán, Estanislao

Njeumi, Felix

Nofemela, Robert S.

Nogales, Aitor

Nogalski, Maciej

Noguchi, Yoshihiro

Nogueira, Mauricio

Noris, Emanuela

Novacek, Jiri

Novelli, Giuseppe

Novosel, Dinko

Nowotny, Norbert

Nuesch, Jurg

Nüesch, Jürg

Nugen, Sam

Nunberg, Jack

Nunez, Alejandro

Núñez, José Ignacio

O'Connor, Christine

Ode, Hirotaka

Odendall, Charlotte

Oesterle, Paul

Offerdahl, Danielle K.

Ogasawara, Noriko

Ogden, Kristen 
Ogembo, Javier

Ogino, Tomoaki

Ohshima, Takayuki

Oka, Tomoichiro

Okabayashi, Tamaki

Okamoto, Hiroaki

Okamoto, Shigefumi

Okayama, Akihiko

Okazaki, Katsunori

Oksanen, Hanna

Olagoke, Olusola

Olds, Cassandra L.

Olech, Monika

Olejniczak, Marta

Olejnik-Schmidt, Agnieszka

Olety, Balaji

Oliver, JoAnne

Olson, Kenneth

Ong, David S.Y.

Onomoto, Koji

Oomens, Antonius (Tom)

Opekun, Antone Robert

Orchard, Robert

Orlova, Elena V.

Orłowska, Anna

Ornelles, David

Orrú, Christina

Ortega, Joseph Thomas

Ortego, Javier

Oscar D., Salomón

Osna, Natalia

Osolodkin, Dmitry

OSSIBOFF, Robert J.

Ottmann, Michèle

Otulak-Kozieł, Katarzyna

Ouwendijk, Werner J. D.

Øynebråten, Inger

Ozawa, Makoto

Ozbun, Michelle

Pacenti, Monia

Pacheco, Beatriz

Pacioni, Carlo

Paczesny, Jan

Padhi, Bhaja Krushna

Paes, Marciano Viana

Paeshuyse, Jan

Pagán, Israel

Page-Karjian, Annie

Paillart, Jean Christophe

Paillot, Romain

Paixão, Enny S.
Pajkrt, Dasja

Palamara, Anna Teresa

Palinski, Rachel

Palmenberg, Ann C.

Palukaitis, Peter

Pandey, Krishan K.

Pandit, Aridaman

Panfil, Amanda

Panganiban, Antonito

Panicz, Remigiusz

Pantůček, Roman

Papin, James F.

Pappu, Hanu

Paquin-Proulx, Dominic

Paradkar, Prasad

Paran, Nir

Parcells, Mark

Parissi, Vincent

Park, Eujin

Park, Junsoo

Parks, Griffith

Parolin, Cristina

Parra, Gabriel

Parreira, Ricardo

Parrish, Colin

Parsons, Matthew

Pascal, Steven M.

Pascalis, Hervé

Pasdeloup, David

Paskaleva, Elena

Passaes, Caroline Pereira Bittencourt

Passler, Thomas

Pastorek, Jaromir

Patel, Trushar R.

Pathak, Vinay K.

Patil, Basavaprabhu

Patterson, Steven

Patterson-West, Jennifer

Paulauskas, Algimantas

Paupy, Christophe

Pavelko, Kevin D.

Pavesi, Angelo

Pavlov, Konstantin

Pawlak, Aleksandra

Paxton, Robert

Payne, Susan

Pazgier, Marzena

Pearson, Angela

PECHEUR, Eve

Peci, Adriana

Pedersen, Finn Skou 
Pedrera, Miriam

Peeters, Ben P. H.

Pegan, Scott D.

Pei, Jingjing

Pei, Yonggang

Peitsch, Manuel Claude

Pelchat, Martin

Pelka, Peter

Pena, Lindomar José

Penades, Jose

Peng, Chen

Penna, Claudia

Pénzes, Judit

Perdoni, Federica

Pereira, Carla

Pereira, Roberto

Perelson, Alan

Pérez Artés, Encarnación

Perez Sepulveda, Blanca

Perez, Daniel

Pérez, Lester J.

Perez-Sautu, Unai

Persson, Ingmar

Peterlin, Boris

Petraityte-Burneikiene, Rasa

Petráš, Marek

Petrillo, Marco

Petrov, Anton

Petruzziello, Arnolfo

Petry, Klaus

Petrzik, Karel

Pettersson, John

Pettersson, Ulf

Peyambari, Mahtab

Pfaender, Stephanie

Pfaller, Christian

Pfeffer, Martin

Phalen, David

Phan, Tung

Piancatelli, Daniela

Piantadosi, Anne

Piazza, Rocco

Pichat, Pierre

Pierangeli, Alessandra

Pietersen, Gerhard

Pietrancosta, Nicolas

Pijlman, Gorben

Pikula, Anna

Pilalas, Dimitrios

Pilotto, Mariana

Pincus, Seth
Pingarilho, Marta

Pintel, David

Pinto, Amelia K.

Pinto, Rosa M

Pipas, James M.

Pires, Diana

Pischke, Sven

Pistello, Mauro

Pittau, Marco

Pizzorno, Marie

Plattet, Philippe

Pleckaityte, Milda

Plemper, Richard

Pleshakova, Tatyana O.

Pöhlmann, Stefan

Pokorski, Mieczyslaw

Polak, Mirosław Paweł

Polonis, Victoria

Polyak, Stephen

Polz-Dacewicz, Małgorzata

Pomar, Leó

Pompon, Julien

Poncet, Didier

Ponraj, Prabakaran

Ponsero, Alise J.

Ponz, Fernando

Pop, Tudor Lucian

Popham, Holly J.

Possee, Robert

Potapov, Sergey A.

Poteat, Tonia

Poterszman, Arnaud

Potokar, Maja

Poulet, Hervé

Poulicard, Nils

Pouliopoulos, Antonis

Poulson, Rebecca

Powers, Ann

Powis, Simon John

Prabakaran, Mookkan

Pradines, Bruno

Prevelige Jr., Peter E.

Preziuso, Silvia

Price, Patricia

Prischi, Filippo

Priyamvada, Lalita

Prodělalová, Jana

Prokhorov, Nikolai

Prosperi, Alice

Proverbio, Daniela

Puchol, Sandra Martínez 
Puertas, Maria Carmen

Puff, Christina

Puig-Barberà, Juan

Pullan, Steven T.

Punga, Tanel

Puray-Chavez, Maritza N.

Qin, Zhiqiang

Qiu, Hua-Ji

Qu, Bingqian

Qu, Feng

Quackenbush, Sandra

Quadeer, Ahmed A.

Quéré, Pascale

Quesada, Ernesto

Quigley, Bonnie L.

Quiñones-Mateu, Miguel E.

Rabalski, Lukasz

Racine, Trina

Radke, Jay $\mathrm{R}$

Radlinska, Monika

Radoshitzky, Sheli R.

Ragan, Izabela

Raghavan, Sudhir

Ragonnert-Cronin, Manon

Ragonnet-Cronin, Manon

Ragupathy, Viswanath

Rahman, Motiur

Rai, Devendra Kumar

Rainey, Stephanie

Rajão, Daniela S.

Rajarapu, Swapna Priya

Rajarathnam, Krishna

Rajko-Nenow, Paulina

Rakonjac, Jasna

Rakotondrafara, Aurelie

Ramanathan, Palaniappan

Ramette, Alban

Ramirez, Hugo

Ranadheera, Senaka

Ranawade, Ayush

Randazzo, Walter

Rappocciolo, Giovanna

Rashed, Arash

Rastelli, Eugenio

Rathinam, Navanietha Krishnaraj

Rathore, Abhay PS

Ratmann, Oliver

Rausch, Jason W.

Ravelonandro, Michel

Ray, Ratna

Rayner, Jonathan
Razzuoli, Elisabetta

Read, Andrew

Rebollo, Rita

Redd, Andrew

Reddy Bolla, Jani

Reddy, Sanjay

Reddy, Vijay

Reddy, Vishwanatha R.A.P.

Redwood, Alec

Regla-Nava, Jose A.

Regoes, Roland

Reguera, Juan

Regunath, Hariharan

Reichel, Michael P.

Reid, Scott

Reid, St Patrick

Reid, Steven

Reid, William

Reina, Ramsés

Reineke, Lucas

Reis, Ana

Ren, Ping

Resa-Infante, Patricia

Rety, Stephane

Reuscher, Carina

Revill, Peter

Reyes-Sandoval, Arturo

Reynolds, Mary

Richardson, Julia

Richman, Douglas D.

Richner, Justin

Richner, Justin M.

Richt, Juergen

Riedel, Christiane

Rieder, Elizabeth

Riera, Marta

Rigling, Daniel

Riley, Kasandra J.

Rim, John Hoon

Rimstad, Espen

Rinder, Monika

Risch, Martin

Rissanen, Ilona

Rittschof, Clare

Rivas, Carmen

Riveiro-Barciela, Mar

Rivero-Juárez, Antonio

Rizvanov, Albert

Robert, Philippe

Roberts, John

Robinson, Chris 
Robinson, Karl

Robinson, Sally R.

Roby, Justin A.

Rockett, Rebecca J

Rockman, Steven

Rodo, Carlota

Rodriguez, Fernando

Rodríguez, Fernando

Rodriguez-Andres, Julio

Rodríguez-Calleja, Jose María

Rodríguez-Díaz, Jesús

Rodriguez-Valera, Francisco

Rogier, Bodewes

Rohrmann, George F

Romalde, Jesús L.

Romano, Patricia Silvia

Romerio, Fabio

Ronca, Shannon

Rong, Chan Kuan

Rong, Lijun

Root, Michael J.

Roques, Pierre

Rosa, Bruce A.

Rosa, Cristina

Rose, Karrie

Rosenthal, Maria

Ross, Perran

Rossetto, Cyprian C.

Rossi, Gianpaolo

Rossier, Ombeline

Rost, Gergely

Rostal, Melinda

Rothenberger, Sylvia

Rothenburg, Stefan

Rotondo, John Cahrles

Roubalova, Katerina

Rouillé, Yves

Rousso, Itay

Routh, Andrew

Routhu, Nanda Kishore

Roux, Simon

Rowland-Jones, Sarah

Rowley, Paul Andrew

Royo, Luis J.

Rozek, Wojciech

Ruaro, Barbara

Rückert, Claudia

Rudd, Penny

Rudra, Arnab

Rueppell, Olav

Ruggiero, Giuseppina
Ruggli, Nicolas

Ruigrok, Rob W.H.

Ruiz-Garcia, Ana Belen

Ruiz-Garcia, Leticia

Rumbou, Artemis

Ruml, Tomas

Rumlová, Michaela

Rusanen, Juuso

Rusconi, Stefano

Russell, Rod

Rut, Wioletta

Ryabov, Eugene

Rybka, Jakub Dalibor

Rynda-Apple, Agnieszka

Ryndock, Eric

Ryu, Sangryeol

Saad, Jamil

Sacco, Randy E.

Sadaoka, Tomohiko

Saelens, Xavier

Safak, Mahmut

Saha, Ipsita

Sahani, Rajkumar

Sahoo, Malaya Kumar

Saitoh, Akihiko

Saitou, Naruya

Sakai, Hiroyasu

Sakamaki, Ippei

Sakellariou, Dikaios

Saksela, Kalle

Saláková, Martina

Salama, Sofie R.

Salánki, Katalin

Salata, Cristiano

Saleh, Fatima A.

Salman, M. D.

Salpini, Romina

Salter, Jason D.

Salvato, Maria

Salvetti, Anna

Samarasinghe, Amali

Samiotaki, Martina

Samy, Ahmed

Samy, Ravi

Sanchez, David Jesse

Sanchez, Gloria

Sanchez-Lockhart, Mariano

Sánchez-Madrid, Francisco

Sandro, Rolesu

Sang, Yongming

Sangster, Mark Y. 
Sankale, Jean-Louis

Sanna, Giuseppina

Sano, Teruo

Sansone, Pasquale

Santiago-Rodriguez, Tasha Marie

Santoro, Maria Mercedes

Santos, Ricardo

Santoso, Netty

Sanz Muñoz, Ivan

Sapp, Martin

Sarathy, Vanessa V.

Sargueil, Bruno

Saribaş, Abdullah Sami

Sarid, Ronit

Sariol, Carlos

Sarker, Subir

Sarr, Moussa

Sarute, Nicolas

Satheshkumar, Panayampalli Subbian

Sato, Hironori

Sattentau, Quentin

Sauder, Christian

Saulle, Irma

Sauter, Daniel

Sautto, Giuseppe A.

Savenkov, Eugene

Savic, Vladimir

Saxena, Pooja

Scallan, Martina

Scarborough, Robert J.

Scaturro, Pietro

Schachner, Adena

Schairer, Cynthia

Scharf, Birgit

Schemmerer, Mathias

Scheuermann, Richard H.

Schildgen, Oliver

Schildgen, Olivier

Schmidt, Aaron G.

Schmidt, Peter Thelin

Schmidt, Philip

Schmidtke, Michaela

Schneider-Schaulies, Juergen

Schneidman-Duhovny, Dina

Schnierle, Barbara S.

Schoen, Robert $\mathrm{T}$.

Schols, Dominique

Schommers, Philipp

Schönrich, Günther

Schotta, Gunnar

Schreiber, Michael
Schrier, Rachel

Schroeder, Declan

Schrum, Adam

Schubert, Grit

Schubert, Ulrich

Schuetz, Alexandra

Schuler Faccini, Lavinia

Schultz, David

Schultz, Thomas

Schulz, Claudia

Schumpp, Olivier

Schvoerer, E.

Schwartz, David A.

Schwarz, Anke

Schweizer, Matthias

Sealy, Joshua

Sediva, Anna

Seferovic, Maxim D.

Segalés, Joaquim

Sekiguchi, Satoshi

Sekijima, Masakazu

Seley-Radtke, Katherine

Semino, Carlos

Semler, Bert L.

Sencanski, Milan

Seo, Sang Heui

Serra-Moreno, Ruth

Serwer, Philip

Setny, Piotr

Sewald, Katherina

Sewald, Xaver

Sgouralis, Ioannis

Shackelford, Julia

Shah, Priya

Shahi, Shailesh K.

Shamay, Meir

Sharma, Lokesh

Sharp, Claire

Sharp, Natasha J.

Sharshov, Kirill

Shaw, Andrew

Sheehy, Noreen

Sherer, Nathan

Shestopalov, Alexander M

Shi, Mang

Shiba, Kiyotaka

Shih, Chiaho

Shim, Hyunbo

Shimoda, Michiko

Shimojima, Masayuki

Shimura, Hanako 
Shin, Hyun-Jin

Shin, Ok Sarah

Shinkai, Yoichi

Shioda, Tatsuo

Shiokawa, Mai

Shirai, Jyunsuke

Shirato, Haruko

Shisler, Joanna

Shivanna, Vinay

Shpacovitch, Victoria

Shriner, Susan

Shukla, Deepak

Sicheritz-Pontén, Thomas

Siddappa, Byrareddy

Siddharthan, Venkatraman

Sieben, Christian

Signorini, Lucia

Silva, Ricardo

Silva, Walter

Silveira, Cynthia

Simon, François

Simpson, David J.

Sin, Jon

Sinderewicz, Emilia

Singanalur, Nagendrakumar

Balasubrama-nian

Singh, Gurdeep

Sinigaglia, Alessandro

Sinkins, Steven

Sioofy-Khojine, Amirbabak

Sironen, Tarja

Sistla, Srinivas

Skelly, Deanne

Skurnik, Mikael

Slavcev, Roderick

Śliwińska-Wilczewska, Sylwia

Sloan, Chantel

Sluis-Cremer, Nicolas

Smartt, Chelsea T.

Smerdou, Cristian

Smith, Bruce

Smith, Donald

Smith, Doug

Smith, Gregory

Smith, Jessica

Smith, Lauren M.

Smith, Thomas

Smith, Todd G.

Smithgall, Thomas E.

Smodiš Škerl, Maja

Smura, Teemu
Snoeck, Chantal J.

Snow, Jonathan W.

Söderberg-Naucler, Cecilia

Söderlund-Venermo, Maria

Sohn, Seonghyang

Sokoloski, Kevin

Sokolova, Olga S.

Solana, Rafael

Solis, Morgane

Sollai, Giorgia

Solmaz, Sozanne

Solovyev, Andrey

Sompuram, Seshi

Song, Jianxun

Song, Min-Suk

Song, Moon Jung

Sooryanarain, Harini

Soriano, Vicente

Soudeyns, Hugo

Sousa, Angela

Souza, Menira

Sozzi, Enrica

Spada, Eva

Sparger, Elizabeth

Spathis, Aris T.

Spatz, Stephen

Speck, Peter G.

Spengler, Jessica R.

Spiegel, Martin

Spiro, David J.

Sridhar, Siddharth

Stadejek, Tomasz

Stafford, Graham P.

Stainton, Daisy

Stamminger, Thomas

Stanifer, Megan L.

Stanton, James B.

Stapleford, Kenneth

Stapleford, Kenneth A.

Stapleton, Jack

Stavolone, Livia

Stavrou, Spyridon

Steel, Laura F.

Steger, Gerhard

Steger, Gertrud

Steinbach, Falko

Steinmann, Eike

Stepanova, Ekaterina

Stephens, Edward

Sternberg-Lewerin, Susanna 
Stevanovic, Jevrosima

Steyer, Dr. Andrej

Stobart, Christopher

Stone, Daniel

Stoye, Jonathan Paul

Strand, Micheline K.

Strecker, Thomas

Strong, Jim

Strutt, Tara M.

Stuart, Patrick

Stubenrauch, Frank

Stukelj, Marina

Su, Weiheng

Suárez, Nicolás M.

Subbiah, Jeeva

Subr, Zdeno

Šubr, Zdeno

Sucena, Élio

Suenaga, Hikaru

Suffredini, Elisabetta

Sukumaran, Sunitha

Sullivan, Brian M.

Sullivan, Chris

Sulzenbacher, Gerlind

Summers, Michael

Sun, Caijun

Sun, Haiyan

Sun, Xiangjie

Sun, Xiaoming

Surapathrudu, Kanakala

Susi, Petri

Sutton, Richard

Sutummaporn, Kripitch

Suzuki, Tohru

Svircev, Antonet

Sweeney, Trevor

Swetnam, Daniele M.

Świętoń, Edyta

Switzer, William

Szakal, Evelin

Szczepankowska, Agnieszka K.

Sztanke, Krzysztof

Szymczak, Aleksandra

Tada, Rui

Tagad, Harichandra D.

Tai, Wanbo

Tai, William Chi-Shing

Tajima, Motoshi

Takada, Ayato

Takahashi, Tomoko

Takano, Tomomi
Takeda, Shigeki

Takemura, Masaharu

Takeuchi, Rikiya

Takeuchi, Yasuhiro

Takimoto, Toru

Takizawa, Naoki

Talker, Stephanie

Tamba, Marco

Tamori, Akihiro

Tan, Wenbin

Tanaka, Yoshikazu

Tandon, Ritesh

Tang, Qiyi

Taniguchi, Koki

Taniguchi, Satoshi

Tao, Pan

Tarasova, Ol'Ga A.

Tarlinton, Rachael

Taskinen, Sara

Tate, Michelle

Tate, Shin-Ichi

Tatsuya, Kato

Tattersall, Peter

Taube, Stefan

Tauriainen, Sisko

Taus, Naomi

Tavanti, Francesco

Taylor, Adam

Taylor, Ian

Te Velthuis, Aartjan

Tedbury, Philip

Telenitsky, Alice

Tempera, Italo

Tempesta, Maria

Tenenbaum, Tobias

Teng, Lee Lee

Teramoto, Tadahisa

Teras, Riho

Terio, Valentina

Terranova, Corrado

Terryn, Sanne

Teschke, Carolyn

Tessarz, Peter

Testoni, Barbara

Thali, Markus

Thanki, Anisha Mahendra

Theuns, Sebastiaan

Thiebes, Anja Lena

Thiry, Etienne

Thomas, John C.

Thomas, Julie 
Thompson, Andrew J.

Thompson, Jeremy

Thwaites, Ryan S.

Tian, Yanping

Tietjen, Ian

Tikoo, Suresh K.

Timofeev, Vladimir I.

Tischer, Karsten

Toborek, Michal

Todt, Daniel

Toffan, Anna

Tognon, Mauro

Tohma, Kentaro

Tollenaere, Charlotte

Tolstrup, Martin

Tonelli, Michele

Tonetti, Michela G.

Tong, Shuping

Toniolo, Antonio Q.

Tonni, Gabriele

Toplak, Ivan

Torcia, Maria Gabriella

Torre, Marialuisa

Torres, Alice

Tortorella, Domenico

Torzewska, Agnieszka

Toth, Karoly

Toubanaki, Dimitra

Toussaint, Ariane

Touzé, Antoine

Trabaud, Mary-Anne

Trapp, Sascha

Trapp-Fragnet, Laëtitia

Travis, John

Traylor-Knowles, Nikki

Treder, Krzysztof

Tremblay, Nicolas

Tripp, Ralph A.

Trobaugh, Derek

Troyer, Ryan

Trubl, Gary

Truncaitè, Lidija

Truniger, Verónica

Trus, Ivan

Tsai, Chi-Wei

Tsai, Jih-Jin

Tsai, Ming-Han

Tsetsarkin, Konstantin A.

Tso, For Yue

Tsourkas, Philippos

Tsuge, Hideaki
Tsukamoto, Tetsuo

Tsukiyama-Kohara, Kyoko

Tsumoto, Kanta

$\mathrm{Tu}$, Thomas

Tucci, Joseph

Tullman-Ercek, Danielle

Tully, Damien

Tuma, Roman

Tumban, Ebenezer

Tun, Mya Myat Ngwe

Tungadi, Trisna

Tuomivirta, Tero

Turchi, Chiara

Turina, Massimo

Turnbull, Matthew W.

Turner, Dann

Twarock, Reidun

Tworowski, Dmitry

Tyagi, Mudit

Tyrrell, D Lorne

Ucciferri, Claudio

Ueda, Keiji

Uehara-Ichiki, Tamaki

Ugaki, Masashi

Ulaeto, David

Ulbert, Sebastian

Ulrich, Rainer

Ulrich, Reiner

Umasuthan, Navaneethaiyer

Unzai, Satoru

Upadhyay, Chitra

Upadhyay, Sandeep

Upton, Chris

Urayama, Shunichi

Urbanelli, Lorena

Urra, José Miguel

Usmani, Shariq

Usui, Tatsufumi

Uversky, Vladimir

V Ivanov, Alexander

Vaira, Anna Maria

Vales-Gomez, Mar

Valiakos, George

Valkenburg, Sophie A.

Valles, Steven

Van Baalen, Minus

Van Borm, Steven

Van Bortel, Wim

Van De Klundert, Maarten

Van De Vijver, David AMC

Van Den Hoogen, Bernadette 
Van Den Hurk, Andrew

Van Den Wollenberg, Diana J. M.

Van Der Hoek, Lia

Van Der Kuyl, Antoinette

Van Der Poel, Wim

Van Der Sluis, Renée M.

Van Doorslaer, Koenraad

Van Engelenburg, Schuyler

Van Etten, James

Van Gils, Marit J.

Van Lint, Carine

Van Marle, Guido

Van Raaij, Mark

Van Rijn, Piet

Van Santen, Vicky

VandeWoude, Sue

Vaney, Marie-Christine

Vanham, Guido

Vanlandingham, Dana

Vanpouille, Christophe

Varallyay, Eva

Varjak, Margus

Varsani, Arvind

Vassaux, Georges

Vasse, Marc

Vassilaki, Niki

Vassilakos, Nikon

Vázquez-Domínguez, Evaristo

Vecchio, Eleonora

Velasco, Leonardo

Velasco-Villa, Andrés

Venuprasad, Poojary

Verchot, Jeanmarie

Vernick, Kenneth D.

Vérollet, Christel

Verrier, Eloi R

Viana, Mafalda

Vidana Mateo, Beatriz

Viejo-Borbolla, Abel

Vijayan, Madhuvanthi

Vilcek, Stefan

Vilibić-Čavlek, Tatjana

Vimalanathan, Selvarani

Vinje, Jan

Viranaicken, Wildriss

Visalli, Robert J.

Visseaux, Benoit

Vitour, Damien

Vivet-Boudou, Valérie

Vlasova, Anastasia N.

Vogels, Chantal
Vogt, Peter K.

Volchkova, Valentina

Volle, Romain

Volz, Asisa

Von Dobschuetz, Sophie

Von Laer, Dorothee

Vranken, Wim

$\mathrm{Vu}$, Luan

Vujadinovic, Marija

Vuorinen, Tytti

Wadell, Göran

Wagemans, Jeroen

Wakimoto, Hiroaki

Waldorf, Kristana Adams

Walker, Kathleen R

Walker, Peter J.

Walther, Paul

Wan, Hongquan

Wan, William

Wang, Bo

Wang, Chihyang

Wang, Ching-Ho

Wang, Chin-Tien

Wang, David

Wang, Fun-In

Wang, Hao-Ching

Wang, HURNG-YI

Wang, Jin

Wang, Jiongwei

Wang, Juexin

Wang, Jun

Wang, Leyi

Wang, Ling

Wang, Nianshuang

Wang, Richard

Wang, Robert Y.-L.

Wang, Xiaohong

Wang, Xiuqin

Wang, Ying

Wang, Zhengqiang

Wang, Ziwen

Wang, Zongjie

Warner, Nadia

Warrell, Mary

Warrilow, David

Wasniewski, Marine

Watanabe, Satoru

Watanabe, Shumpei

Watanabe, Tokiko

Webster, Craig

Weger, Stefan 
Wegrzyn, Alicja

Wegrzyn, Grzegorz

Węgrzyn, Grzegorz

Wei, Dongqing

Wei, Hongping

Wei, Yonglong

Weidner-Glunde, Magdalena

Weinbauer, Markus

Weiser, Rebecca

Weissenhorn, Winfried

Weitzman, Matthew

Welch, Stephen R

Welsh, Michael

Wen, Chiu-Ming

Wenping, Qiu

Wertheim, Joel

Westman, Mark

Weyenbergh, Johan Van

Weynberg, Karen

Whitby, Denise

White, John

White, Judith

White, Kirsten

White, Peter A.

White, Simon

Whitford, Paul C

Whitney, James B.

Whitney, John

Wichgers Schreur, Paul J.

Wigdahl, Brian

Wilke, Andre

Wilkes, Rebecca P.

Willett, Brian

Wilson, Angus

Wilson, Carolyn A.

Wilson, Van G.

Wilson, William

Wilson, William C.

Wilusz, Jeff

Winkler, Michael E.

Winter, Deborah Rachelle

Wira, Charles

Wise, Annabel G.

Wise, Emma L.

Witkowski, Wojciech

Wold, William S.M.

Wolf, Steven

Wölfel, Roman

Wollebo, Hassen S.

Wong, Grace

Wong, Richard W.
Wong, Sek-Man

Wood, Britta

Workman, Aspen M.

Woźniakowski, Grzegorz

Wright, Daniel

Wu, Fang-Tzy

$\mathrm{Wu}$, Guanghui

Wu, I-Chin

$\mathrm{Wu}$, Jianyong

$\mathrm{Wu}$, Suh-Chin

$\mathrm{Wu}$, Wenxin

$\mathrm{Wu}$, Xueling

$\mathrm{Wu}$, Yanling

Wu, Yuntao

Wylie, Kristine

Xavier, Catarina

Xia, Ningshao

Xia, Yuchen

Xian, Yuejiao

Xiao, Yongli

Xie, Deyu

Xie, Jiatao

Xie, Jiexiong

Xin, Yin

Xu, Jian

$\mathrm{Xu}$, Pei

Xu, Wenxing

Xue, Guangai

Yamamoto, Masato

Yamamoto, Takehisa

Yamanaka, Atsushi

Yamaoka, Satoko

Yamauchi, Yohei

Yamayoshi, Seiya

Yanase, Tohru

Yang, Hung-Chih

Yang, Kui

Yang, Siyoung

Yang, Xiuling

Yao, Yongxiu

Yasuike, Motoshige

Yau, Sheree

Ye, Xiaohua

Yeager, Meredith

Yeh, Hsin-Hung

Yehl, Kevin

Yellapu, Nanda Kumar

Yen, Pei-Shi

Yin, John

Yin, Li

Yin, Renfu 
Yin, Zhimin

Ylösmäki, Erkko

Yolande, Richard

Yoldi, Miguel Julián Martínez

Yoneyama, Mitsutoshi

Yoo, Ji Young

Yoo, So Young

Yoon, Hachung

Yoon, Sun-Woo

Yoshida, Eric

Yoshimatsu, Kumiko

Yost, Christian Con

Youk, Sungsu

Young, Howard A.

Young, Ryland

Yount, Jacob S.

Yousef, Ahmed

Yu, Jingyou

Yu, Xiao-Fang

Yu, Xue-Jie

Yuan, Hao

Yuan, Shuofeng

Yuchun, Du

Yue, Xiao-Guang

Yuen, Lilly

Yuh, Chiou-Hwa

Yuill, Thomas

Yura, Yoshiaki

Zachou, Kalliopi

Zago, Miriam

Zaid, Ali

Zakhartchouk, Alexander

Zamperin, Gianpiero

Zanella, Isabella

Zanier, Katia

Zanusso, Gianluigi

Zapata, Juan

Zecchin, Bianca

Zeev-Ben-Mordehai, Tzviya

Zehender, Gianguglielmo

Zeichner, Steven L.

Zell, Roland

Zeltins, Andris

Zeman, Petr

Zeng, Lanying

Zhang, Bo

Zhang, Dayi

Zhang, Gongyi

Zhang, Hong-Yu
Zhang, Huanmin

Zhang, Qiong

Zhang, Rui

Zhang, Shuo

Zhang, Wenli

Zhang, Yinfeng

Zhang, Yong

Zhang, Zhenfeng

Zhang, Zhidong

Zhang, Zhifang

Zhao, Jianjun

Zhao, Jun

Zhao, Qinjian

Zhao, Xue Zhi

Zheng, Du-Ping

Zheng, Wenshu

Zheng, Xuexing

Zheng, Zhenhua

Zheng, Zhi-Ming

Zheng, Zi-Zheng

Zhirnov, Oleg

Zhong, Zhaohua

Zhou, Aimin

Zhou, Dongming

Zhou, Grace G

Zhou, Heshan Sam

Zhou, Peng

Zhou, Shuntai

Zhu, James

Zhu, Jian

Zhu, Qiyun

Zhu, Xueyong

Zidovec Lepej, Snjezana

Zienius, Dainius

Zimmer, Gert

Zimmerman, Jeffrey

Zmora, Pawel

Żok, Tomasz

Zoll, Jan

Zolnik, Christine P.

Zou, Wei

Zumbrun, Elizabeth

Žunec, Renata

Zúñiga, Manuel

Zuñiga, Sonia

Zuriaga, Elena

Züst, Roland

Zwick, Michael B. 